\title{
Alternate Day Statin Dosing for Women with Previous Statin Intolerance Margo Minissian ${ }^{1}$, Talya Waldman ${ }^{1}$, Chrisandra Shufelt ${ }^{1}$, Megha Agarwal ${ }^{4}$, Puja K. Mehta ${ }^{2}$, Galen Cook-Wiens ${ }^{1}$, Jo Ann Eastwood $^{3}$ and Noel Bairey Merz ${ }^{1}$
}

${ }^{1}$ Barbra Streisand Women's Heart Center, Cedars-Sinai Heart Institute, Cedars-Sinai Medical Center, Los Angeles, California, United States ${ }^{2}$ Emory Women's Heart Center, Atlanta, Georgia, United States

${ }^{3}$ University of Los Angeles, School of Nursing, Los Angeles, California, United States

${ }^{4}$ Ventura Cardiology Consultants, Ventura, California, United States

\begin{abstract}
Background: HMG-CoA reductase inhibitors (statins) are efficacious for treatment and prevention of cardiovascular disease (CVD). Adherence is problematic due to side effects which prevent high risk patients from receiving benefit from statins.

Aim: The aim of this study was to evaluate the effectiveness of a nurse practitioner (NP) alternate statin dosing protocol for the reduction of total cholesterol (TC) and low-density lipoprotein (LDL-C).

Methods: 15 women who had the inability to tolerate daily dosing were analyzed using a novel NP protocol for initiation and maintenance of alternate dosing utilizing atorvastatin, pravastatin, or rosuvastatin. Patients were started on twice weekly statin dosing for 4 weeks then titrated up one dose per week as tolerated. While on alternate statin dosing, fasting lipid levels were ordered and obtained by chart review. We compared baseline and alternate day dosing by Wilcoxon signed rank test.

Results: We evaluated 15 female patients with a mean age of $62 \pm 14$ years and BMI $26 \pm 5 \mathrm{~kg} / \mathrm{m}^{2}$. Overall, $60 \%$ had CVD, $60 \%$ had hypertension, $7 \%$ had diabetes, and $47 \%$ had a history of smoking but currently non-smokers. Prior to the study, all women were intolerant to 2 or more statins. Utilizing the alternate dosing NP protocol, we observed a $32 \%$ reduction in LDL-C, to a mean of $84 \pm 39 \mathrm{mg} / \mathrm{dl}$ compared to the baseline mean of $134 \pm 49 \mathrm{mg} / \mathrm{dl}(\mathrm{p}<0.001)$ and a $20 \%$ reduction of TC $(\mathrm{p}<0.0001)$.

Conclusions: Among women with statin intolerance, an NP statin alternate dosing protocol is effective in reducing TC and LDL-C levels. Further studies using this protocol are warranted in larger statin intolerant populations.
\end{abstract}

\section{Introduction}

Cardiovascular disease (CVD) is the single largest killer of women, [1] and more women than men die each year despite advancements in life-saving therapies [2]. In fact, patients with a total blood cholesterol level greater than $200 \mathrm{mg} / \mathrm{dL}$ have a two-fold risk of developing CVD [3]. 3-Hydroxy-3-methylglutaryl-coenzyme A (HMG-CoA) reductase inhibitors (statins) are currently the most effective treatment for lowering total cholesterol (TC), calculated low density lipoprotein (LDL-C), and CVD risk [4].

Despite diagnosis and treatment in women, CVD death reduction has not matched that of men [5]. Women have less compliance to statin medication than men [6]. Poor adherence to statins may be related to medication costs and side effects. In randomized clinical trials, myalgias are reported in $1 \%$ to $5 \%$ of patients in statin and placebo groups; [7] however, observational data with non-selected patients show higher rates of myalgias up to $20 \%$ [8]. We tested a nurse practitioner (NP) guided protocol which is aimed at reducing TC and LDL-C in women with prior statin intolerance due to myalgias.

\section{Methods}

The Barbra Streisand Women's Heart Center at the Cedars-Sinai Heart Institute is a tertiary chest pain center where we recruited 15 consecutive women with a medical indication for statin therapy and a history of myalgias. For patients with multiple baseline TC and LDL-C measurements in chart review, the highest prior LDL-C was selected. Myalgic pain was defined as benign, moderate to severe myopathic symptoms such as: myalgia, myositis and myopathy but not rhabdomyolysis, based on the ACC/AHA/NHLBI guidelines [8].

\section{Publication History:}

Received: September 29, 2016

Accepted: December 01, 2016

Published: December 03, 2016

\section{Keywords:}

Statin intolerance, Myalgias, Statin alternate dosing, Women pain related to statin use. Other etiologies of myopathic pain were ruled out at the patient's clinical visit.The study was approved by the Cedars-Sinai Medical Center ethical review board.

We defined statin intolerance as the inability to tolerate daily dosing of at least one prior statin. Our novel NP protocol for alternate dosing statin regimen used pravastatin, atorvastatin or rosuvastatin for the reduction of TC and LDL-C, selected by individualized statin and dosing depending on the patient's medication formulary, need for LDL reduction and the Cytochrome (CYP) pathway to avoid medication interactions.

Patients were initially started on twice weekly statin dosing on Mondays and Thursdays for 4 weeks then titrated up one additional dose per week as tolerated until either daily dosing was achieved or the patient experiences myalgic pain. If myalgias developed, the patient was taken back to the immediately prior tolerated dosing regimen. Fasting baseline and follow-up lipid levels were obtained by chart review.

"Corresponding Author: Margo Minissian, PhDc, ACNP, Barbra Streisand Women's Heart Center, Cedars-Sinai Heart Institute, 127 San Vicente AHSP suite A9306, Los Angeles, CA 90048, United States; E-mail: minissianm@cshs.org

Citation: Minissian M, Waldman T, Shufelt C, Agarwal M, Mehta PK, et al. (2016) Alternate Day Statin Dosing for Women with Previous Statin Intolerance. Int J Nurs Clin Pract 3: 212. doi: https://doi.org/10.15344/2394-4978/2016/212

Copyright: (c) 2016 Minissian et al. This is an open-access article distributed under the terms of the Creative Commons Attribution License, which permits unrestricted use, distribution, and reproduction in any medium, provided the original author and source are credited. 
Citation: Minissian M, Waldman T, Shufelt C, Agarwal M, Mehta PK, et al. (2016) Alternate Day Statin Dosing for Women with Previous Statin Intolerance. Int J Nurs Clin Pract 3: 212. doi: https://doi.org/10.15344/2394-4978/2016/212

Page 2 of 3

\section{Ethics statement}

All patients were informed of the study and provided a written informed consent. The Cedars-Sinai Institutional Review Board (CSMC IRB) approved the study (CR00006479/Pro0019454). The investigation conforms to the principles outlined in the Declaration of Helsinki.

\section{Statistical analysis}

All statistical analysis was performed using SAS (ver. 9.2; The SAS Institute, Cary, NC). Means and standard deviations (SD) were reported for all continuous variables. Wilcoxon signed rank test was performed on the data.

\section{Results}

We evaluated and treated 15 consecutive female patients with a mean age of $62 \pm 14$ years (Table 1). Prior to initiating the alternate dosing NP protocol, 93\% were intolerant to 2 or more statins (simvastatin, pravastatin, atorvastatin, or rosuvastatin) with associated myalgias.

\begin{tabular}{|l|l|}
\hline \multicolumn{2}{|c|}{ Baseline Characteristics } \\
\hline Age (Year \pm SD) & $62 \pm 14$ \\
\hline Hypertensive & $60 \%$ \\
\hline CAD & $60 \%$ \\
\hline Diabetics & $7 \%$ \\
\hline History of Smoking & $47 \%$ \\
\hline Table 1: Baseline Demographics. \\
\hline
\end{tabular}

In the NP alternate dosing statin protocol, the most common regimen was rosuvastatin $5 \mathrm{mg}$ with twice weekly dosing, Mondays and Thursdays (Figure 1). These patients were not able to increase their days of the week after 4 weeks and maintained a twice weekly regimen. Three of fifteen $(20 \%)$ patients needed to reduce their regimen to once weekly dosing. The remaining 6 patients were able to progress to 3-4 day per week dosing with either atorvastatin or rosuvastatin (Figure 1).
One patient was taking rosuvastatin $5 \mathrm{mg}$ with three days per week dosing which included Mondays, Wednesdays and Fridays. She needed to take an every-other week drug holiday to avoid severe myalgias. Patients were followed up 4-6 weeks after the initiation of the NP protocol.

Utilizing the alternate dosing NP protocol, we observed significant reductions in TC and LDL-C compared to their prior highest TC and LDL-C (Figure 2).

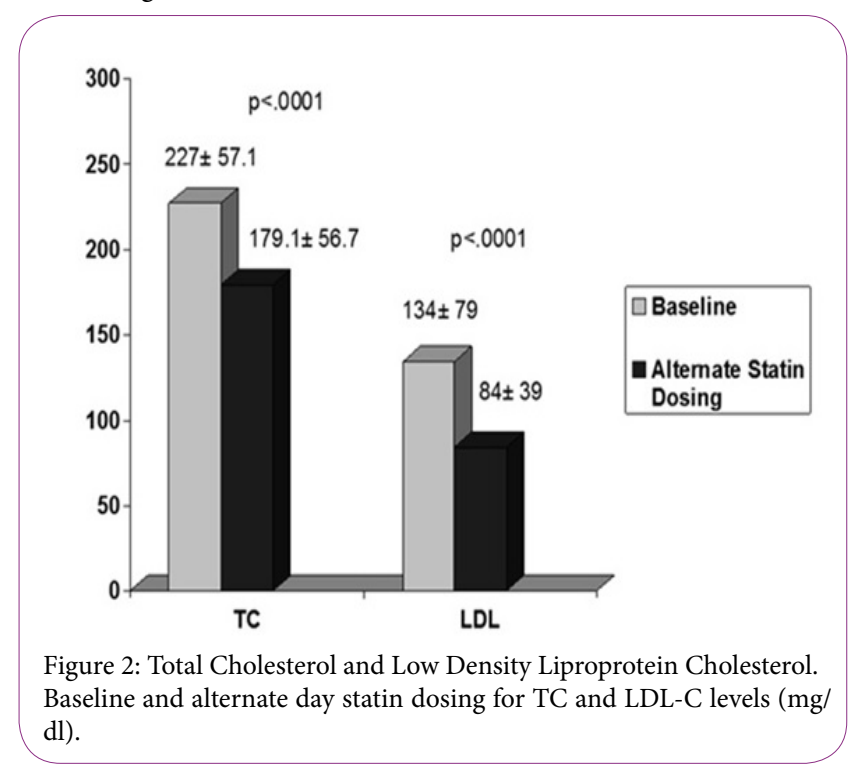

\section{Discussion}

Among women with a history of daily dose statin intolerance, an NP guided protocol is effective for reducing TC and LDL-C levels. This pilot study is the first to evaluate 3 different statins with multiple alternate dosing regimens. Although different statins have different LDL-C lowering capabilities, this study suggests that an alternate statin regimen may be superior to no statin for lowering TC and LDL-C. The data would further suggest that our NP alternate dosing

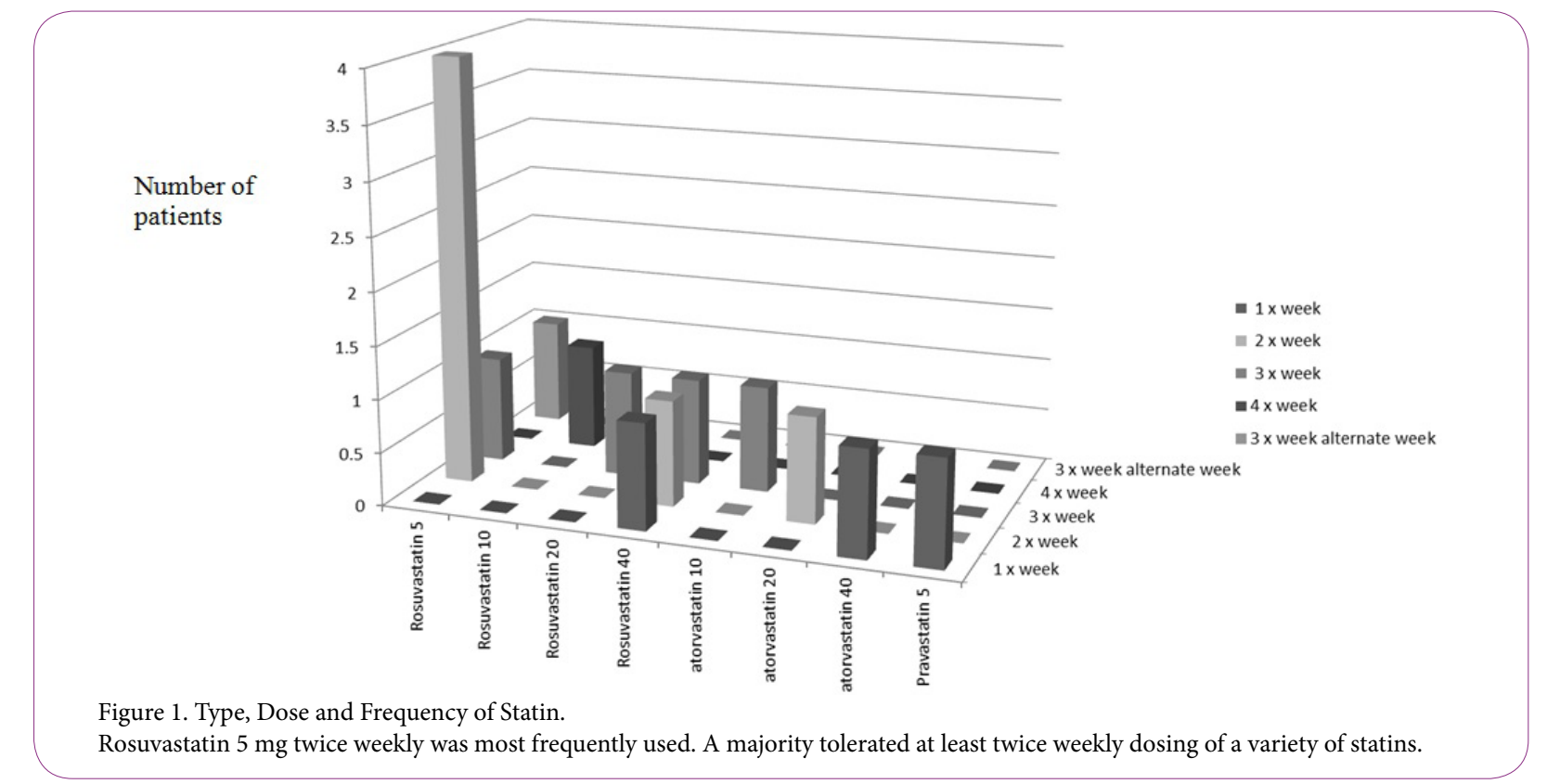


Citation: Minissian M, Waldman T, Shufelt C, Agarwal M, Mehta PK, et al. (2016) Alternate Day Statin Dosing for Women with Previous Statin Intolerance. Int J Nurs Clin Pract 3: 212. doi: https://doi.org/10.15344/2394-4978/2016/212

Page 3 of 3

protocol may be an effective strategy and should be considered before classifying a patient as statin intolerant.

Our results are consistent with previous studies and support the hypothesis that alternate statin dosing is a successful strategy for treating prior statin intolerant patients. Our most common regimen was rosuvastatin $5 \mathrm{mg}$ with twice weekly dosing, Mondays and Thursdays. Rosuvastatin alternate day dosing is desirable because of its generous half-life of 19 hours [9] and its potent LDL-C lowering capabilities. Gardarla, Kearns and Thompson [10] published similar results when they evaluated 40 patients with prior statin myalgias who were placed on twice weekly rosuvastatin $(5 \mathrm{mg}$ and $10 \mathrm{mg}$ ) either alone or in addition to other lipid-lowering agents. They showed an LDL-C reduction of $26 \%$ and TC reduction of $19 \%$, which is similar to our results [10]. Backes et al performed a retrospective analysis on patients taking rosuvastatin $(2.5 \mathrm{mg}, 5 \mathrm{mg}, 10 \mathrm{mg})$ in 51 patients. Their mean LDL-C decreased by $34.5 \%$ on an average dose of rosuvastatin $5.6 \mathrm{mg}$ per $\mathrm{mg} / \mathrm{dl}$ which also correlates with our findings [11]. In addition, once-a-week dosing using rosuvastatin (2.5 mg-20 $\mathrm{mg}$ ) also appears to be beneficial. Ruisinger et al. demonstrated a $23 \%$ reduction in LDL-C and 17\% reduction in TC during a mean follow up of 4 months [12]. Their results are also consistent with our data in which $3 / 15$ (20\%) tolerated only the once-weekly regimens.

The Alternate Day Versus Daily dosing of Atorvastatin Study showed similar results with atorvastatin $(10-20 \mathrm{mg})$ alternate day dosing compared to daily dosing. This double blind, placebo-controlled design evaluated 35 patients who met National Cholesterol Education Program (NCEP) Adult Treatment Panel (ATP) III guidelines for statin therapy. Although higher doses were used on alternate day, their results suggest that alternate dosing can produce similar reduction in LDL-C comparable to that of daily administration of atorvastatin [13]

\section{Limitations}

There are several limitations to this pilot study. Our data is observational and not prospectively obtained, without placebo controls or blinding. Lipid levels were not standardized to timing and myalgias were based on patient report and/ or clinical chart review. Furthermore, the sample size is small. Nevertheless, there is association among the percentage of TC and LDL-C lipid lowering and statin dosing. There was also bias in the selection of the TC and LDL-C with selecting the prior highest result. A larger prospective and placebo controlled trial would be needed to evaluate if an NP guided alternate dosing protocol could truly alter cardiovascular outcomes through cholesterol lowering.

\section{Conclusion}

Among women with a history of daily dose statin intolerance, an $\mathrm{NP}$ alternate dosing protocol is effective in reducing TC and LDL-C levels. Further studies using this NP alternative statin dosing protocol are warranted in larger populations who are statin intolerant to determine whether an alternate dosing protocol leads to increased longer term statin adherence and whether this impacts the rates of cardiovascular disease outcomes in women.

\section{Implications for Practice}

This alternate statin dosing protocol for higher risk patients who are intolerant to normal statin dosing due to complications and sideeffects may:

\section{- $\quad$ Lower TC and LDL-C \\ - Help to reduce myalgic pain in women with statin intolerance \\ - Provide an alternative treatment than not implementing any statin use.}

\section{Competing Interests}

The authors declare that they have no competing interests.

\section{Funding}

This work was supported by contracts from the National Heart, Lung and Blood Institutes:1F31NR015725-01, N01-HV-68161, N01HV-68162, N01-HV-68163, N01-HV-68164, grants U0164829, U01 HL649141, U01 HL649241, T32HL69751, 1R03AG032631 from the National Institute on Aging, GCRC grant MO1-RR00425 from the National Center for Research Resources and grants from the Gustavus and Louis Pfeiffer Research Foundation, Danville, NJ, The Women's Guild of Cedars-Sinai Medical Center, Los Angeles, CA, The Ladies Hospital Aid Society of Western Pennsylvania, Pittsburgh, PA, and QMED, Inc., Laurence Harbor, NJ, the Edythe L. Broad Women's Heart Research Fellowship, Cedars-Sinai Medical Center, Los Angeles, California, and the Barbra Streisand Women's Cardiovascular Research and Education Program, Cedars-Sinai Medical Center, Los Angeles.

\section{References}

1. Heron M, Hoyert DL, Murphy SL, Xu J, Kochanek KD, et al. (2009) Deaths: final data for 2006. National vital statistics reports : from the Centers for Disease Control and Prevention, National Center for Health Statistics, National Vital Statistics System 57: 1-134.

2. Mosca L, Benjamin EJ, Berra K, Bezanson JL, Dolor RJ, Lloyd-Jones $\mathrm{DM}$, et al. (2011) Effectiveness-based guidelines for the prevention of cardiovascular disease in women--2011 update: a guideline from the american heart association. Circulation 123: 1243-1262.

3. America's Cholesterol Burden January 30,2012.

4. Grundy SM, Cleeman JI, Merz CN, Brewer HB Jr, Clark LT, et al. (2004) Implications of recent clinical trials for the National Cholesterol Education Program Adult Treatment Panel III guidelines. Circulation 13: 227-239.

5. Bellasi A, Raggi P, Merz CN, Shaw LJ (2007) New insights into ischemic heart disease in women. Cleve Clin J Med 74: 585-594.

6. Ellis JJ, Erickson SR, Stevenson JG, Bernstein SJ, Stiles RA, Fendrick AM (2004) Suboptimal statin adherence and discontinuation in primary and secondary prevention populations. J Gen Intern Med 19: 638-645.

7. Sewright KA, Clarkson PM, Thompson PD (2007) Statin myopathy: incidence, risk factors, and pathophysiology. Curr Atheroscler Rep 9: 389396.

8. Pasternak RC, Smith SC Jr., Bairey-Merz CN, Grundy SM, Cleeman JI, et al. (2002) ACC/AHA/NHLBI clinical advisory on the use and safety of statins. Journal of the American College of Cardiology 40: 567-72.

9. Lopez LM (2005) Rosuvastatin: a high-potency HMG-CoA reductase inhibitor. J Am Pharm Assoc (2003) 45: 503-513.

10. Gadarla M, Kearns AK, Thompson PD (2008) Efficacy of rosuvastatin (5 $\mathrm{mg}$ and $10 \mathrm{mg}$ ) twice a week in patients intolerant to daily statins. Am J Cardiol 101: 1747-1748.

11. Backes JM, Venero CV, Gibson CA, Ruisinger JF, Howard PA, et al. (2008) Effectiveness and tolerability of every-other-day rosuvastatin dosing in patients with prior statin intolerance. Ann Pharmacother 42: 341-346.

12. Ruisinger JF, Backes JM, Gibson CA, Moriarty PM (2009) Once-a-week rosuvastatin $(2.5$ to $20 \mathrm{mg})$ in patients with a previous statin intolerance. Am J Cardiol 103: 393-394.

13. Matalka MS, Ravnan MC, Deedwania PC (2002) Is alternate daily dose of atorvastatin effective in treating patients with hyperlipidemia? The Alternate Day Versus Daily Dosing of Atorvastatin Study (ADDAS). Am Heart J 144: 674-677. 